\title{
Adjunctive mild hypothermia therapy to primary percutaneous coronary intervention in patients with ST segment elevation myocardial infarction complicated with cardiogenic shock: A pilot feasibility study
}

\author{
Alex Blatt ${ }^{1 *}$, Gabby Atalya Elbaz-Greener ${ }^{1 *}$, Avi Mizrachi ${ }^{2}$, Ziad J'bara ${ }^{1}$, \\ Tali Taraboulos ${ }^{2}$, Ilia Litovchik ${ }^{1}$, Zvi Vered, Sa'ar Minha ${ }^{1}$ \\ ${ }^{1}$ Cardiology Department, Assaf Harofeh, Medical Center, Tel Aviv University, Tel Aviv, Israel \\ ${ }^{2}$ Assaf Harofeh, Medical Center, Tel Aviv University, Tel Aviv, Israel
}

\begin{abstract}
Background: Despite successful primary reperfusion therapy, patients may develop large myocardial infarction related in part to reperfusion injury. Induction of mild therapeutic hypothermia (TH) applied in patients has demonstrated beneficial effect in reducing reperfusion injury. The aim of the study was to evaluate the feasibility and safety of adjunctive mild TH to primary percutaneous coronary intervention (PPCI) in patients with acute ST elevation myocardial infarction (STEMI) complicated with cardiogenic shock (CS).

Methods: We conducted a prospective single center, open label, historical control study. Patients presenting with STEMI and CS despite maximal support therapy scheduled for PPCI were included. Death was defined as primary endpoint. Secondary outcomes included: TH adverse effect - such as fever, refractory hypotension and arrhythmias, infarct size measured by area under the curve of biomarkers.

Results: Eight consecutive patients were prospectively enrolled (TH group). Thirteen clinically similar patients identified from our database, admitted over 2 years, comprised the historical control group (control group). In the hypothermia group, the mortality was $50 \%$ compared with $46 \%$ in the control group. There was no difference in the secondary outcomes.

Conclusions: TH as adjunctive therapy in STEMI patients complicated with CS is feasible and safe. Based on these preliminary observations there appears to be no significant clinical advantage to this form of therapy. (Cardiol J 2015; 22, 3: 285-289)
\end{abstract}

Key words: therapeutic hypothermia, ST elevation myocardial infarction, cardiogenic shock

\section{Introduction}

Cardiogenic shock (CS) affects approximately $5 \%$ to $7 \%$ of patients with myocardial infarction (MI) and is the leading cause of death among hospitalized patients with MI, with mortality approaching $50 \%$ despite urgent reperfusion therapy, optimal medical care, and intra-aortic balloon support [1].

Address for correspondence: Alex Blatt, MD, Cardiology Department, Assaf Harofeh, Medical Center, Tel Aviv University, Tel Aviv, Israel, e-mail: alexb@asaf.health.gov.il

*These authors contributed equally

Received: 25.06.2014 Accepted: 24.08.2014 
Therapeutic hypothermia (TH) is the standard of care in the treatment for out-of-hospital ventricular tachycardia/fibrillation arrest. The potential beneficiary neuroprotective effect is by reducing reperfusion injury [2]. Theoretically, the cooling properties of $\mathrm{TH}$ that protect the brain and promote recovery after cardiac arrest may have similar effects on other vital organs, such as the infracted heart and other injured organs due to CS. The cardioprotective effects of TH have been reported for over 30 years [3]. The benefit of mild TH as a treatment for acute MI (AMI) has now been demonstrated in several animal models [4] and small to intermediate scale feasibility randomized clinical trials [5-7]. A small reduction in temperature $\left(2-4^{\circ} \mathrm{C}\right)$ may result in a profound reduction effect on infarct size [8]. Experimental studies have shown that there is a strong correlation between infarct size and body temperature and the size of ischemic risk zone, which becomes necrotic dependent on temperature. In humans, the adjunctive intravascular cooling before mechanical reperfusion in uncomplicated AMI appears to be safe and may reduce infarct size without significant delay in the door to balloon time [9]. Recently, it has been proposed that CS is a systemic illness that needs systemic treatment, such as TH [10]. There are yet no clinical studies investigating prospectively the effect of $\mathrm{TH}$ in patients with $\mathrm{CS}$ following AMI.

The aim of this study was to evaluate the safety and feasibility of non-invasive cooling induction as adjuvant therapy in patients with a ST-elevation MI (STEMI) complicated with CS scheduled to undergo primary percutaneous coronary intervention (PPCI).

\section{Methods}

\section{Ethics}

The present study was performed in accordance with the Declaration of Helsinki. The study protocol was approved by the local Ethics Committee. Since all included patients had been sedated and invasively ventilated, the authors were exempt from obtaining prospective written informed consent. Close family members received detailed explanation about the study aims and protocol. After the patients' recuperation and rehabilitation, the study protocol was given to all surviving patients.

\section{Study population}

It was a single center, prospective, open label, historical control study. Patients were enrolled to test the feasibility and safety of an infusion of cold normal saline at $4^{\circ} \mathrm{C}$ at a high rate, alongside to a designated hypothermia suit, as an adjunct therapy in patients with an acute coronary syndrome complicated with pump failure CS eligible for PPCI. Men and women between 18 and 75 years of age who presented with an anterior or inferior STEMI with ST-segment elevation of $0.2 \mathrm{mV}$ in 2 or more anatomically contiguous leads or ST depression of $0.3 \mathrm{mV}$ or more in $\mathrm{V}_{3}-\mathrm{V}_{4}$ and a duration of symptoms of $<12 \mathrm{~h}$ were included. Pump failure CS was defined as persistent hypotension, systolic pressure $<90 \mathrm{~mm} \mathrm{Hg}$ despite fluids and catecholamine infusion. Patients with cardiac arrest, known congestive heart failure, significant valve disease, CS related to any mechanical complication (rupture free wall, ventricular septal defect, tamponade, acute mitral regurgitation, significant right ventricle infarction), end-stage kidney disease or hepatic failure, recent stroke, coagulopathy or pregnancy, or absence of maximal support therapy, were excluded from the study.

\section{Protocol}

Eligible patients were prospectively assigned to $\mathrm{TH}$ group. Mild $\mathrm{TH}\left(32-34^{\circ} \mathrm{C}\right)$ was applied for $12 \mathrm{~h}$; non-invasive induction was obtained by combining an IV infusion of normal saline at $4^{\circ} \mathrm{C}$ at a $5-15 \mathrm{~mL} / \mathrm{kg} / \mathrm{h}$ rate, alongside to a designated hypothermia suit. After induction, hypothermia was maintained by the suit only. Body core temperature was monitored independently using 3 separate probes. Re-warming was initiated after $12 \mathrm{~h}$ of hypothermia at a rate of $0.3^{\circ} \mathrm{C}$ per hour. All patients were sedated with midazolam and a muscle relaxant in order to prevent shivering. Once normal body temperature was regained, sedation was halted. After induction of hypothermia started, coronary angiogram was performed. Loading doses of $300 \mathrm{mg}$ of aspirin and $600 \mathrm{mg}$ of clopidogrel were given to all patients before cardiac catheterization. Patients underwent PCI according to current standard of practice.

The historical control group was selected based on similar inclusion criteria.

The primary outcome measure was death at 30 days. The secondary outcome measure was MI size, assessed by cardiac markers (troponin $\mathrm{T}$ and creatinine kinase $[\mathrm{CK}]$ ) and $\mathrm{TH}$ related complications such as infection or refractory arrhythmias.

\section{Biochemical markers}

Troponin $\mathrm{T}$ and $\mathrm{CK}$ were sampled on admission to the catheterization laboratory, and at $12 \mathrm{~h}$ and $24 \mathrm{~h}$ after admission. Peak values were defined as 
Table 1. Patients' baseline characteristics.

\begin{tabular}{lccc}
\hline & $\begin{array}{c}\text { Active group } \\
\text { (hypothermia, } \mathbf{n = 8}\end{array}$ & $\begin{array}{c}\text { Control group } \\
\text { (normothermia, } \mathbf{n}=13 \text { ) }\end{array}$ & P \\
\hline Age (median) & $69.6 \pm 7$ & $65.1 \pm 10$ & NS \\
Male & 6 & 9 & NS \\
Hypertension & 5 & 11 & NS \\
Diabetes mellitus & 3 & 6 & NS \\
Dyslipidemia & 6 & 7 & NS \\
Current smoker & 5 & 4 & NS \\
Past myocardial infarction & 3 & 6 & NS \\
Chronic renal failure & 3 & 6 & NS \\
ST elevation myocardial infarction & 7 & 11 & NS \\
Non-ST elevation myocardial infarction & 1 & 2 & NS \\
Anterior myocardial infarction & 7 & 9 & NS \\
Non-anterior myocardial infarction & 1 & 4 & NS \\
Subacute stent thrombosis & 1 & 1 & NS \\
Late stent thrombosis & 0 & 1 & \\
\hline
\end{tabular}

Table 2. Angiographic, procedural and laboratory data.

\begin{tabular}{|c|c|c|c|}
\hline & $\begin{array}{c}\text { Active group } \\
\text { (hypothermia, } n=8 \text { ) }\end{array}$ & $\begin{array}{c}\text { Control group } \\
\text { (normothermia, } n=13 \text { ) }\end{array}$ & $\mathbf{P}$ \\
\hline Door-to-balloon time [min] & $79 \pm 21$ & $86 \pm 26$ & NS \\
\hline Infarct related artery: & & & NS \\
\hline Left main & 2 & 4 & NS \\
\hline Left anterior descending artery & 5 & 6 & NS \\
\hline Right coronary artery & 1 & 3 & NS \\
\hline Non culprit PCI & 4 & 5 & NS \\
\hline Initial TIMI flow: & & & NS \\
\hline $0 / 1$ & 6 & 11 & NS \\
\hline $2 / 3$ & 2 & 2 & NS \\
\hline Successful reperfusion & 8 & 13 & NS \\
\hline Final TIMI flow 3 & 6 & 10 & NS \\
\hline Non reflow phenomenon & 4 & 6 & NS \\
\hline Median peak creatinine kinase (12 h) & $4,200 \pm 424$ & $5,906 \pm 525$ & NS \\
\hline Median creatinine kinase (72 h) & $1,637 \pm 272$ & $2,894 \pm 263$ & NS \\
\hline Area under the curve (72 h) & $7,343 \pm 5,742$ & $14,361 \pm 10,197$ & NS \\
\hline
\end{tabular}

$\mathrm{PCl}$ - percutaneous coronary intervention; TIMI — Thrombolysis in Myocardial Infarction

the highest measured value within $24 \mathrm{~h}$. The area under the curve (AUC) was calculated in each patient.

\section{Statistical analysis}

Standard statistical calculations were used. Comparison between groups was based on the unpaired t-test, statistical significance was defined as $\mathrm{p}<0.05$.

\section{Results}

Twenty-one patients were enrolled into the study: 8 patients prospectively in the TH group and 13 in the historical control group (normothermia group). There were no significant differences in baseline characteristics between the hypothermia and the control groups (Table 1). Laboratory and angiographic data are shown in Table 2. Success- 


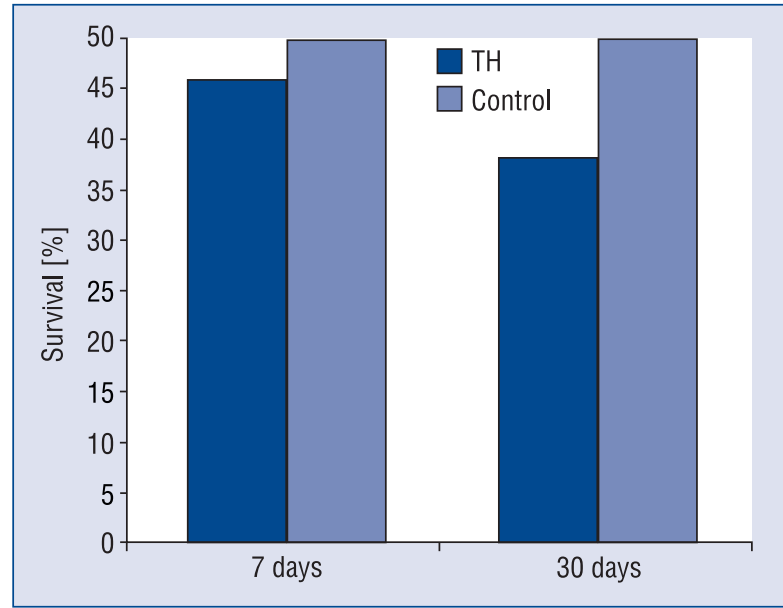

Figure 1. Patients survival at 7 and 30 days; $\mathrm{TH}-$ therapeutic hypothermia

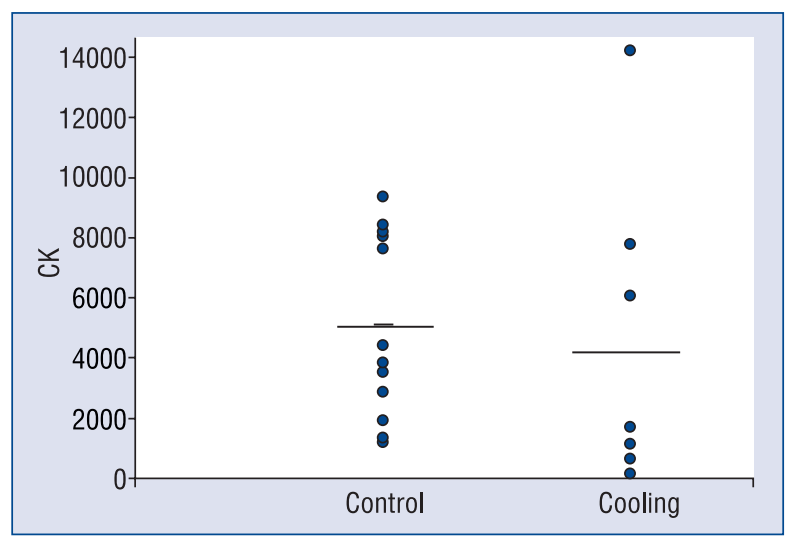

Figure 3. Peak creatinine kinase (CK) levels release.

ful revascularization was achieved in all patients and a stent was inserted in all with Thrombolysis in Myocardial Infarction (TIMI) 3 flow at the end of the procedure.

\section{Hypothermia treatment}

Baseline temperature was similar in the two groups $\left(36.5 \pm 0.6^{\circ} \mathrm{C}\right.$ vs. $36.6 \pm 0.8^{\circ} \mathrm{C}$, $\mathrm{TH}$ vs. control, $\mathrm{p}<0.69)$. Door-to-balloon time was $79 \pm 21 \mathrm{~min}$ in the $\mathrm{TH}$ group and $86 \pm 26 \mathrm{~min}$ in the control group, indicating that induction of hypothermia did not cause any significant delay in time to reperfusion $(\mathrm{p}<0.22)$ In the TH group, at the time of reperfusion, core body temperature was $35 \pm 0.7^{\circ} \mathrm{C}$. The target temperature was achieved $118 \pm 27 \mathrm{~min}$ after induction. The maintenance phase extended by over $12 \mathrm{~h}$ in all 8 patients.

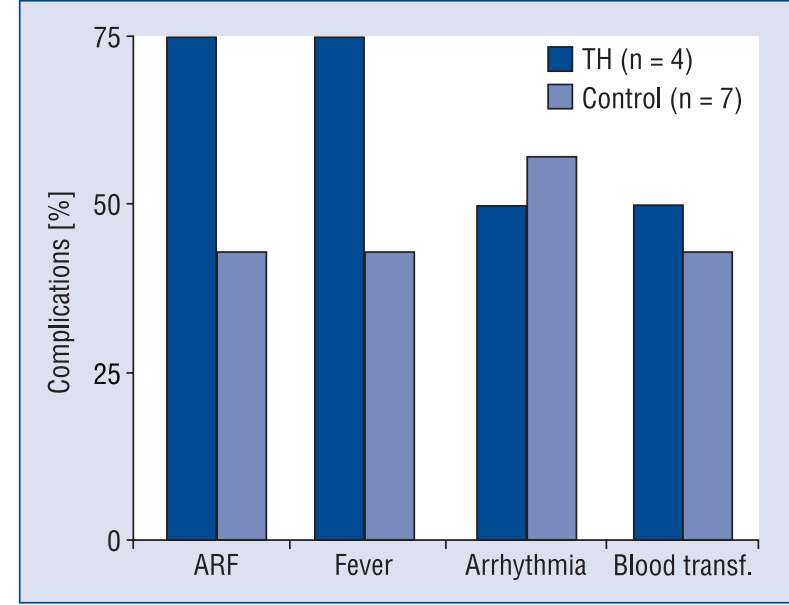

Figure 2. Hospital complications; TH - therapeutic hypothermia; ARF — acute renal failure.

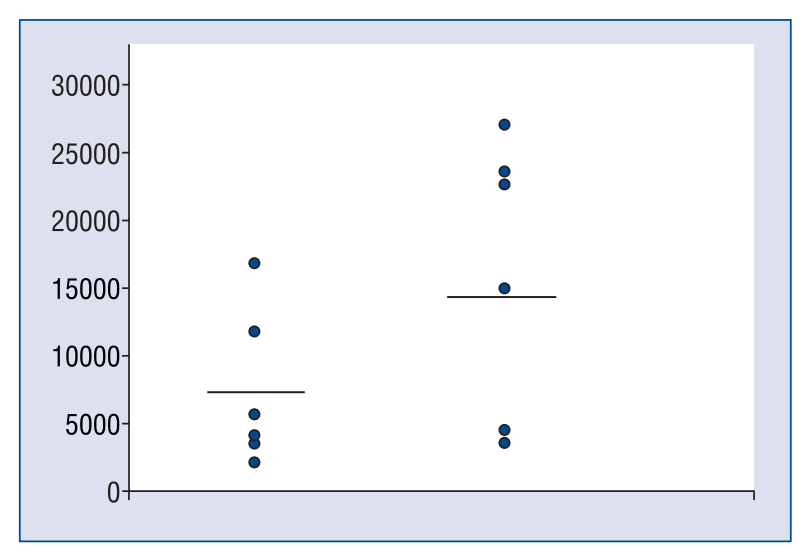

Figure 4. Calculated area under the curve.

\section{Clinical events/safety}

There were no significant differences between the groups with regard to clinical events (Fig. 1). In the TH group, the mortality at 7 days was $50 \%$ compared with $46 \%$ in the control group. The surviving patients in the TH group returned to good functional class at 6 months and 3 of the 4 alive patients had some systolic function recuperation at 3-month echocardiography assessment. There were no differences in the incidence of fever, infection, significant arrhythmia or blood transfusion between the two groups (Fig. 2).

\section{Biochemical markers}

Peak CK (Fig. 3) and troponin T levels did not differ between the groups, although a mild trend toward reduction was observed in the $\mathrm{TH}$ group 
$(4,200 \pm 424$ vs. $5,096 \pm 525 \mathrm{~g} / \mathrm{L}, \mathrm{p}=0.17$ and $8.8 \pm 4.3$ vs. $8.02 \pm 5.4 \mu \mathrm{g} / \mathrm{L}, \mathrm{p}=0.11$, respectively between the TH vs. control). The AUC for total CK calculated only in patients with 5 consecutive measurements, did not show a significant difference between the groups (Fig. 4) either, although a similar trend was observed ( $\mathrm{TH} 7,343 \pm 5,742$ vs. control 14,361 $\pm 10,197, \mathrm{p}=0.08)$.

\section{Discussion}

Cardiogenic shock is a systemic clinical syndrome. Therapeutic strategies with global systemic effects may offer additional benefit over standard cardiac support. TH may potentially offer systemic beneficial physiologic effects, including the probability to improve post-ischemic cardiac function and hemodynamics, decrease myocardial damage, and reduce end-organ injury from prolonged hypoperfusion. Favorable effects of TH have been demonstrated in ischemic animal models of CS by reduction of ischemia/reperfusion injury after urgent revascularization for AMI. In humans, 3 studies showed decreased infarct size in patients with acute non-complicated infarcts treated with intravascular cooling. Clinical experience of $\mathrm{TH}$ in CS is limited. Several case reports and case series of TH in patients with CS, limited to pediatric and adult cardiac surgery patients, have been described. Patients with CS following AMI have not been included.

The present study demonstrates that using a combination of an infusion of cold saline together with a disposable suit to induce mild hypothermia in patients with acute coronary syndrome complicated with pump failure CS is feasible and safe without significant door to balloon delay. No significant difference in clinical event rate was observed between the groups.

\section{Limitations of the study}

The primary aim of this study was to assess the safety and feasibility of hypothermia in AMI complicated with CS. Survival and MI size reduction assessed by cardiac biomarkers was a secondary end point, and even though a significant reduction was not observed, these results should be interpreted with caution due to the small number of patients, the nature of the control group (retrospective, non-consecutive) and infarct size estimated only by biomarkers.

\section{Conclusions}

The results of the present study indicate that $\mathrm{TH}$ as an adjunctive therapy in STEMI patients complicated with CS, applied before reperfusion, is clinically feasible and safe without significant delay in time to reperfusion. Larger clinical studies are needed to evaluate the potential clinical efficacy of this approach to treat CS in the setting of AMI.

\section{Conflict of interest: None declared}

\section{References}

1. Babaev A, Frederick PD, Pasta DJ, Every N, Sichrovsky T, Hochman JS. Trends in management and outcomes of patients with acute myocardial infarction complicated by cardiogenic shock. JAMA, 2005; 294: 448-454.

2. Hazinski MF, Nolan JP, Billi JE et al. Part 1: Executive summary: 2010 International consensus on cardiopulmonary resuscitation and emergency cardiovascular care science with treatment recommendations. Circulation 2010; 122: S250-S275.

3. Abendschein D, Tacker W, Babbs C. Protection of ischemic myocardium by whole-body hypothermia after coronary artery occlusion in dogs. Am Heart J, 1978; 96: 7722-7780.

4. Hale S, Kloner R. Myocardial temperature reduction attenuates necrosis after prolonged ischemia in rabbits. Cardiovasc Res, 1998; 40: 502-507.

5. Gotberg M, Olivecrona G, Koul S. A pilot study of rapid cooling by cold saline and endovascular cooling before reperfusion in patients with ST-elevation myocardial infarction. Circ Cardiovasc Interv, 2010; 3: 400-407.

6. Kandsari DE, Chu A, Brodie BR et al. Feasibility of endovascular cooling as an adjunct to primary percutaneous coronary intervention: Results of the LOWTEMP pilot study. Am J Cardiol, 2004; 93: 636-639.

7. Erlinge D, Gotberg M, Lang I et al. Rapid Endovascular Catheter Core Cooling combined with cold saline as an Adjunct to Percutaneous Coronary Intervention For the Treatment of Acute Myocardial Infarction (The CHILL-MI trial). J Am Coll Cardiol, 2014; 63: 1857-1865.

8. Sharon L. Hale and Robert A. Kloner mild hypothermia as a cardioprotective approach for acute myocardial infarction: Laboratory to clinical application. J Cardiovasc Pharmacol Ther, 2011; 16: 131-139.

9. Erlinge D, Gotberg M, Grines C. A pooled analysis of the effect of endovascular cooling on infarct size in patients with ST-elevation myocardial infarction. EuroIntervention, 2013; 8: 1435-1440.

10. Stegman BM, Newby LK, Hochman JS, Ohman EM. Post-myocardial infarction cardiogenic shock is a systemic illness in need of systemic treatment: is therapeutic hypothermia one possibility? J Am Coll Cardiol, 2012; 59: 644-647. 